\title{
Review
}

\section{Pediatric Acupuncture: A Review of Clinical Research}

\author{
Jeffrey I. Gold ${ }^{1}$, Colette D. Nicolaou ${ }^{2}$, Katharine A. Belmont ${ }^{2}$, Aaron R. Katz ${ }^{3}$, \\ Daniel M. Benaron ${ }^{3}$ and Wendy $\mathrm{Yu}^{4}$
}

\author{
${ }^{1}$ Keck School of Medicine, University of Southern California, ${ }^{2}$ Department of Anesthesiology Critical Care \\ Medicine, Childrens Hospital Los Angeles, ${ }^{3}$ Department of Hematology/Oncology, Childrens Hospital Los Angeles \\ and ${ }^{4}$ Eastern Center for Complementary Medicine, Inc., Los Angeles, CA, USA
}

\begin{abstract}
Practiced in China for more than 2000 years, acupuncture has recently gained increased attention in the United States as an alternative treatment approach for a variety of medical conditions. Despite its growing prevalence and anecdotal reports of success among pediatric populations, few empirically based studies have assessed the efficacy of acupuncture for children and adolescents. This article presents a review of the current literature, including a systematic appraisal of the methodological value of each study and a discussion of potential benefits and adverse effects of acupuncture. While acupuncture holds great promise as a treatment modality for diverse pediatric conditions, a significant amount of additional research is necessary to establish an empirical basis for the incorporation of acupuncture into standard care.
\end{abstract}

Keywords: acupuncture - pediatrics

\section{Introduction}

Over the past several decades, the use of complementary and alternative medicine (CAM) has increased in the general population. Between 1990 and 1997, the percentage of American patients using alternative therapies grew considerably, from $\sim 34$ to $42 \%$ (1). In 1999, more than $50 \%$ of physicians surveyed in the United States, Canada, Israel and England reported recommending CAM to their patients (1). Accordingly, many insurance providers, including managed care organizations, now offer reimbursement for CAM therapies $(2,3)$.

Among pediatric populations, CAM interventions such as homeopathy, naturopathy, acupuncture, chiropractic, herbal supplements, vitamin and mineral supplementation, macrobiotics and dietary modifications are reportedly

For reprints and all correspondence: Jeffrey I. Gold, Ph.D., Childrens Hospital Los Angeles, Department of Anesthesiology Critical Care Medicine, Keck School of Medicine, University of Southern California, 4650 Sunset Blvd. MS \#12, Los Angeles, CA 90027-6062, USA.

Tel: +1-323-361-6341; Fax: +1-323-361-1022;

E-mail: jgold@chla.usc.edu used by $2 \%$ to $20-30 \%$ of patients (4-6). Higher rates have been reported among children with specific medical conditions, such as pediatric cancer, rheumatoid arthritis and cystic fibrosis, where $30-70 \%$ of patients employ CAM therapies $(7,8)$.

In response to its increasing popularity in both adult and pediatric populations, the National Institutes of Health has allocated a substantial amount of funding to the integration of CAM into mainstream medical practice through the creation of the National Center for Complementary and Alternative Medicine (NCCAM). During the 10-year period between 1992 and 2002, NCCAM funding increased by more than $\$ 100$ million, from $\$ 2$ million to \$104.6 million (9). In 2006, NCCAM was allocated \$122.7 million in order to fund CAM research (9).

Within the repertoire of CAM strategies, acupuncture has emerged as a popular method of managing symptoms associated with a variety of medical conditions (10-12). Acupuncture is among the CAM therapies most frequently recommended by internists and family physicians $(10,13-15)$ and is currently practiced in over 140 hospitals in the United States (16). 
Prior to the advent of modern medicine, acupuncture evolved as a primary health care modality for treatment of diverse conditions involving orthopedics, gastroenterology, obstetrics/gynecology and cardiology (17-21). Originating in China, acupuncture has been practiced as a form of bioenergetic healing (i.e. relating to the flow and transformation of energy in and between living organisms and between living organisms and their environment) for over 2000 years (22). Although there are several variations in its practice, acupuncture is based upon the notion of energy or 'Qi' (pronounced 'Chee'), which is distributed throughout the body via a complex network of channels, or 'meridians' (23). Specific acupoints, which serve as target points in order to restore altered or obstructed Qi, can be found at precise positions along each meridian and are accessed using a variety of methods, the most common of which is dry needles $(23,24)$.

In response to the widespread use of acupuncture throughout history, recent research has sought to critically examine the efficacy of acupuncture as a treatment for diverse conditions. In adult populations, research suggests beneficial effects for conditions including fibromyalgia $(25,26)$, dyspnea (27), nausea and vomiting $(28,29)$, tinnitus $(30)$, stroke rehabilitation (31), angina (31), menopausal symptoms (31), dysmenorrhea (31), anxiety (32-34) and pain management (35). As the adult literature continues to determine the efficacy of acupuncture, the utilization and awareness of acupuncture in the treatment of pediatric conditions is also gaining increased attention. An examination of 43 pain clinics in North American children's hospitals revealed that approximately one-third offered acupuncture services (36). Most research, which has focused on prevalence rates and acceptability, suggests that a large number of children are being referred for acupuncture, that the notion of receiving this type of alternative treatment is generally acceptable to their parents and that they are happy with the experience overall (37). This appears to contradict the mainstream notion that children are afraid of needles and that parents may not want to subject them to additional stress and pain $(37,38)$.

Despite the high prevalence of CAM usage in children, few empirically-based studies have examined the effectiveness of most CAM therapies in a pediatric population. A literature review of the web-based search engines PubMed, PsychInfo and Medline was conducted using the following search terminology: pediatric acupuncture, acupuncture and children, acupuncture and adolescents, pediatric CAM, CAM and children, and CAM and adolescents. All available studies addressing pediatric acupuncture were chosen for inclusion, with the exception of studies with poor methodological quality (e.g. poorly defined outcomes, sample size $<10$, etc.). This review will examine pediatric acupuncture across specific disease states, followed by a global assessment of design and methodological limitations commonly noted within this literature base. Contraindications associated with acupuncture and potential future directions will also be discussed.

\section{Studies Assessing Acupuncture for Pediatric Populations}

\section{Chronic Pain}

Kemper and colleagues (37) conducted a retrospective qualitative examination of 47 children and adolescents, aged 5-20 years, referred for acupuncture for a variety of chronic pain conditions. Treatment strategies included needles $(98 \%$ of children), moxibustion or heat $(85 \%)$, cupping (26\%) and magnets $(26 \%)$. Results revealed that $67 \%$ of patients and $60 \%$ of parents described the acupuncture experiences as positive or pleasant. In addition, $70 \%$ of patients and $59 \%$ of parents reported that treatment was helpful in alleviating pain. Despite methodological limitations, this study revealed a high degree of acceptability among patients and parents, as well as painreducing benefits associated with acupuncture.

A more recent study by Zeltzer and colleagues (39) examined the acceptability and feasibility of a CAM intervention among a pediatric sample with chronic pain. Thirty-three children, aged 6-18 years, were referred for a combined treatment of acupuncture and hypnosis. The acupuncture portion of treatment included six weekly sessions involving 6-15 needles placed at individuallydetermined acupoints, based on traditionally recognized methods. Participants also underwent progressive muscle relaxation and guided imagery exercises for $20 \mathrm{~min}$. Findings revealed that $29 \%$ and $16 \%$ of participants had prior experience with acupuncture and hypnosis, respectively. Although parents with previous experience anticipated lower effectiveness, experience and anticipated effectiveness ratings were not associated with treatment outcome. Anticipatory anxiety and pain ratings taken during each session revealed that both variables significantly declined as treatment sessions progressed. In addition, both parent and child reported current pain ratings decreased from baseline to post-treatment on the Varni-Thompson Pediatric Pain Questionnaire (40). Almost half the participants experienced a $50 \%$ reduction in current pain ratings after the intervention. There was also a significant increase in functioning according to both child and parents reports, such that there was less pain-related interference in various arenas, including physical activity and activities with friends. Depression scores on the Children's Depression Inventory (41) did not significantly change over the course of treatment, however, over $90 \%$ of the sample did not endorse clinically significant depression prior to the intervention. According to the State-Trait Anxiety Inventory for 
Children (STAIC) - State Version (42), there was a nonsignificant decline in reported levels of anxiety over the course of treatment.

In two related studies, Lin and colleagues investigated the benefits of acupuncture for children and adolescents with chronic pain (43) and chronic fatigue (44). Fiftythree children and adolescents, aged 2-18 years, with a variety of painful conditions, reported significant reductions in visual analog scale (VAS) ratings of pain between pre- and post-acupuncture measurements. The average duration of the 'acupuncture effect', however, was 3.0 days. In addition, the method of determining effect length was unclear, and seemed to suggest only temporary benefits associated with acupuncture as well as the need for continual treatment for long-term improvement. Eight adolescents, aged 11-18 years, with chronic fatigue syndrome reported similar reductions in average pain ratings, as well as temporary reductions in fatigue and an increase in energy after each treatment; however, the authors do not note whether these improvements are statistically significant. A more thorough presentation of the methods and statistical analysis is needed to draw accurate conclusions.

Hand acupuncture has been studied among a sample of 106 children, aged 3-20 years, who presented to a general pediatric practice with a variety of acute and chronic conditions (45). Participants were encouraged to continue standard of care medical treatments (e.g. antibiotics, steroids) in addition to acupuncture therapy. Based on child- and parent-reports and clinical observations, $96 \%$ of participants experienced improvement in their symptoms, defined as complete resolution of pain or $\mathrm{a} \geq 50 \%$ reduction in pain magnitude. Approximately $70 \%$ of children noted improvements in $<3$ days, however most chronic symptoms improved within 1-2 weeks. As the author does not clearly define outcome measurement procedures or statistical findings, this study is difficult to interpret.

\section{Pediatric Migraine}

Although several studies have examined the effectiveness of acupuncture as treatment for adult headaches and migraines, as well as a recent review suggesting "promising results' $(46,47)$, there has only been one examination within a pediatric population. Twenty-two children, aged $7-15$ years, with migraines were randomized to receive either acupuncture or placebo acupuncture (48). In the acupuncture group, needles were inserted subdermally, in accordance with Traditional Chinese Medicine. The placebo group received needle insertions within the stratum corneum, a more superficial skin layer. Weekly frequency-intensity questionnaires revealed a significant decrease in migraine frequency and intensity among participants in the acupuncture group, however, no such decline was observed within the placebo group. Findings from this investigation are particularly limited due to the exclusion of participants who used daily medications, a highly prevalent practice among this population.

\section{Procedural Stress}

Within the pediatric literature, one randomized, controlled study was conducted among a premature infant population to examine the potential stress-reducing effects of acupuncture during an ophthalmologic examination for retinopathy of prematurity (ROP) (49). Twenty-two infants were randomized to an acupuncture group, which was administered a single session of electroacupuncture during the ROP examination, or a control group. Saliva samples were taken prior to electrode placement and $15 \mathrm{~min}$ after the ROP was completed to examine cortisol concentration, a marker of stress. Findings revealed that salivary cortisol levels increased among both the treatment and control groups, however, only the increase in the control group was statistically significant. Electrical stimulation was administered at a low frequency and intensity in order to avoid potential adverse events; therefore, the results are limited to low frequency and intensity stimulation. No conclusions can be drawn regarding use of a greater frequency or intensity of stimulation.

\section{Enuresis}

Early studies within the acupuncture literature reveal success rates as high as $98.2 \%$ among enuretic populations; however, these studies poorly define outcome variables and lack methodological empiricism (50-53). More recently, Bower and colleagues (54) performed a review assessing the use of acupuncture for nocturnal enuresis within the pediatric literature. Of the 206 articles examined, only 11 studies met criteria for inclusion. Nevertheless, they found that combined heterogeneous forms of acupuncture, as well as acupuncture combined with Chinese herbal remedies, might reduce the number of wet nights to a greater extent than a single treatment modality. Notably, they did not find a significant difference between acupuncture and antidiuretic medication trials.

Perhaps the most empirically sound study cited in the aforementioned review was a randomized trial investigating laser acupuncture as compared with desmopressin, a commonly used antidiuretic drug (55). The standard treatment group consisted of 20 children, aged 5-16 years, who received intranasal doses of desmopressin for 3 months. Twenty children, aged 5-14 years, were randomized to receive 10-15 acupuncture sessions in which pre-specified regions were targeted with a lowpower laser. Post-treatment assessments at 6 months revealed that $75 \%$ of participants in the desmopressin group were complete responders (at least $90 \%$ reduction of wet nights) and $10 \%$ were partial responders (at least $50 \%$ 
reduction in number of wet nights). Similarly, in the acupuncture group, $65 \%$ of the children remained completely dry at 6 months and $10 \%$ were partial responders. Considering the spontaneous cure rate of $15 \%$ a year among enuretic populations, these findings suggest a role for acupuncture as an alternative treatment option for families.

An earlier study (56) examined 25 children, aged 7-16 years, who had previously been treated for nocturnal enuresis. Children underwent 20 sessions of electroacupuncture, in which pre-selected regions were manually stimulated, and/or stimulated through painless low frequency biphasic pulses. At the 6-month follow-up, five of the children were considered responders $(\geq 90 \%$ reduction in number of wet nights) and six were deemed partial responders ( $\geq 50 \%$ reduction in number of wet nights). Although a majority of children were non-responders at 6 months, $65 \%$ of the sample experienced an increase in dry nights such that the median numbers of dry nights increased from 2.3 to 5.0 dry nights per week.

A more recent study examined 15 children, aged 6-18 years, with nocturnal enuresis (57). Participants received four weekly sessions of acupuncture in pre-selected acupoints. Findings revealed that $20 \%$ of children were deemed cured ( $\geq 90 \%$ reduction in number of wet nights), while another $20 \%$ showed clinical improvement $(\geq 50 \%$ reduction in number of wet nights) immediately after intervention. In addition, at the 2-month follow up, $47 \%$ of the sample was deemed cured. A similar study conducted among 50 children with nocturnal enuresis, aged 9-18 years, found $86 \%$ of participants experienced no bedwetting at a 6-month follow up (58).

In addition, Korean hand acupuncture for children with nocturnal enuresis has been examined in a doubleblind, placebo controlled, crossover study among 26 children, aged 5-13 years (59). A reduction of wet nights was observed under both active and sham Korean hand therapy conditions, however, more children improved while undergoing active treatment. A total of $53 \%$ of participants experienced $90 \%$ dry nights at 3-month follow-up. However, outcome measurements and statistical findings were not clearly defined in this study.

Although pharmacotherapy is highly effective among children with enuresis, parents are often hesitant to initiate pharmacological treatments. Therefore, behavioral strategies, including alarm therapy and bladder stretching exercises, have been employed and often used in conjunction with medication $(60,61)$. As the efficacy of acupuncture continues to be explored, its use as an alternative treatment option for enuresis among children and adolescents appears to be gaining validity.

\section{Constipation}

Only one study has investigated the use of acupuncture for the treatment of childhood constipation (62). Twenty-seven children, aged 3-13 years, who had been experiencing constipation for at least 6 months were initially enrolled in the study, however, 10 children were excluded due to poor compliance. Fifteen children, aged 2-14 years, who did not experience any gastrointestinal difficulties, comprised the control group. Participants initially received five weekly placebo acupuncture session in which needles were inserted into the stratum corneum near the actual acupoints. Following the placebo intervention, participants underwent 10 weekly acupuncture sessions through subdermal needling. Both males and females showed a similar significant increase in number of bowel movements per week after 10 weeks of acupuncture, however, females tended to improve more quickly. In fact, female participants began to display improvements after the initial 5 -week placebo acupuncture trial. This study holds promise for the role of acupuncture in the treatment of pediatric constipation, yet more research is clearly needed to replicate these findings.

\section{Epilepsy}

The Cochrane Collaboration recently published a review of acupuncture research in the treatment of epilepsy (63), however only one study examined a pediatric sample. Specifically, Xiong (64) published a study in China that assessed 64 boys, aged 1-17 years, with epilepsy. Participants were randomized into one of three groups: fixed point acupuncture and Chinese herbs, Chinese herbs alone, or carbamaxepine alone. Findings revealed a $<50 \%$ reduction in seizure frequency in $90 \%, 80 \%$ and $73 \%$ of participants in the three treatment groups, respectively. However, this did not represent a statistically significant difference in improvement between groups. Furthermore, acupuncture plus herb treatment did not statistically differ in terms of the length of seizures or EEG normalization as compared with the herb or carbamazepine treatment alone. In conjunction with these non-significant findings, the Cochrane review concluded that there was no strong evidence for acupuncture as a treatment for epilepsy.

\section{Allergies}

Despite the fact that only one randomized trial has been conducted in the adult literature, $5 \%$ of adults with allergic rhinitis in the United States have reportedly used acupuncture to alleviate their symptoms $(65,66)$. Similarly, only one randomized study has been conducted to assess the effects of acupuncture on pediatric allergic rhinitis. Ng and colleagues (67) conducted a double blind, randomized, placebo controlled study among children in a Hong Kong Hospital with persistent allergic rhinitis. Seventy-two children, aged 6-20 years, were assigned to receive acupuncture or sham acupuncture, where needles were inserted only $0.3 \mathrm{~cm}$ into the skin and were not 
rotated as in the active acupuncture protocol, biweekly for 8 weeks. During the 12 weeks following treatment, a significant reduction in daily rhinitis scores was reported among the acupuncture group. Similarly, the acupuncture group had significantly more symptom free days during the treatment and follow-up periods. Visual analog scores also revealed greater immediate improvement among those in the acupuncture group. Laboratory findings revealed that post-treatment $\operatorname{IgE}$ levels, which indicate the presence of an allergic antibody, were significantly elevated in the sham group, but not the acupuncture group, as compared with baseline data.

\section{Neurologic Disability}

Several studies have been conducted among children with neurological disabilities in order to examine the role of acupuncture in alleviating specific maladaptive symptoms $(68,69)$. One such study examined the impact of tongue acupuncture on drooling problems among 10 treatmentresistant children, aged 2-18 years, with various neurological disorders (69). Each child received 30 sessions of daily acupuncture over 6 weeks. Prior to treatment and during each session, the primary caretaker completed a VAS assessing drooling severity (70) and recorded drooling severity and frequency to yield an overall drooling score, as previously validated by Heine, CattoSmith and Reddihough (71). In addition, a blinded research assistant calculated a drooling quotient (72) several times a day. All outcome measures revealed statistically significant improvements over the course of treatment. In addition, 6 month follow-up data suggested that most mothers observed lasting improvements in severity drooling.

A second tongue acupuncture study assessed functional outcomes among a sample of 33 children, aged 3-16 years, with cerebral palsy (73). Twenty-two participants were randomized into the treatment group and received 40 daily sessions of tongue acupuncture over 8 weeks. Eleven participants were initially placed in the control group and received 40 sessions of sham acupuncture in which needles were pressed upon, but not inserted into, specified acupoints. Two weeks after the 40 sham acupuncture sessions, the control group crossed over to receive a course of tongue acupuncture. The Gross Motor Functional Measure [GMFM: (74)] was used to assess changes in gross motor skills and the Pediatric Evaluation of Disability Index [PEDI: (75)] was administered to determine self-care, social and cognitive functioning. The initial control group was administered a third set of measures after receiving the true acupuncture intervention at week 20. Results suggested a significant increase in motor functioning and significant improvements on the mean self-care subscale, the mean mobility subscale and the mean self-care assistance subscale over the course of true acupuncture treatment. No significant differences were detected on the PEDI among the sham acupuncture control group, however, the mean GMFM significantly improved. Paired differences between groups suggested that the mean increase in GMFM scores within the test group was significantly greater than that observed in the control group.

Duncan and colleagues (76) conducted a randomized, controlled study investigating osteopathic manipulations and acupuncture among children, aged 11 months-12 years, with spastic cerebral palsy. Participants were enrolled in either a waitlist control group, which included non-therapeutic time with a volunteer $(n=19)$, an osteopathic manipulation group $(n=23)$, or an acupuncture group in which individually determined and symptom-specific acupoints were employed $(n=19)$. Based on clinical interviews, $91.3 \%$ of parents in the osteopathic group and $100 \%$ of the parents in the acupuncture group reported improvements in their children, while only $12 \%$ of control group parents reported improvements. Improved functioning was frequently described as increased use of legs or hands, more restful sleep, improved mood, more regular bowel movements and decreased muscle stiffness.

\section{Laryngospasm}

Researchers examined the use of acupuncture to prevent laryngospasm, a common side effect of pediatric general anesthesia associated with tracheal extubation (77). After waking from general anesthesia for various surgical procedures, 76 children, aged 1-10 years, were randomly assigned to receive either acupuncture or no treatment. Findings revealed a statistically significant difference in the number of patients who developed post-extubation laryngospasms, such that $5.3 \%$ of the acupuncture group and $23.7 \%$ of the control group evidenced laryngospasm. It is important to note that patients who did develop laryngospasm were immediately treated with acupuncture and blood letting; symptoms resolved within $1 \mathrm{~min}$ of treatment in all patients.

\section{Post-operative Vomiting}

In contrast to the aforementioned conditions, numerous studies have assessed the efficacy of acupuncture for the management of post-operative nausea and vomiting (78-81). An early study by Yentis and Bissonnette (81) examined 90 children, aged 1-16 years, undergoing elective outpatient strabismus surgery. Participants were randomly assigned to one of three groups, including droperidol (82), droperidol plus $5 \mathrm{~min}$ of manually stimulated acupuncture on the P6 acupoint, or acupuncture alone. Acupuncture was administered after anesthesia induction, but prior to surgery. Results revealed no significant differences between the three treatment conditions in terms of total incidence of vomiting or 
vomiting before hospital discharge. However, children in the droperidol group were noted to be significantly more restless post-operatively, as compared with children in the acupuncture alone group.

An additional study among 187 children, aged 7-16 years, examined post-operative nausea and vomiting after general anesthesia and outpatient surgery by employing four study groups: (i) intravenous saline and P6 acupuncture, (ii) droperidol and P6 sham acupuncture, (iii) saline and sham acupuncture and (iv) saline and P6 sham acupuncture (83). Findings revealed a significantly lower incidence of PACU nausea and vomiting in the P6 group as compared with the sham group and the P6 sham group, but not the droperidol group.

Schlager and colleagues (84) made several modifications to earlier methodologies $(81,85)$ in order to test the efficacy of acupuncture for post-operative vomiting in a similar sample of children undergoing strabismus surgery. Their treatment group $(n=25$, mean age $=6.5)$ received Korean hand acupressure, in which a disc is used to apply pressure to the Korean K-K9 acupoint, 30 min prior to the induction of anesthesia. The disc was secured in place for at least $24 \mathrm{~h}$. In addition, this study employed a control group $(n=25$, mean age $=6.5)$, which had tape, but no disc, placed over acupoints in order to simulate treatment. Results indicated a significant difference in the incidence of post-operative vomiting between the two study groups, such that only $20 \%$ of patients in the treatment group, but $68 \%$ of control patients vomited after surgery. In contrast to the Yentis and Bissonette studies $(81,85)$, interventions were administered while children were still awake, prior to the delivery of anesthesia. This strategy coincides with the adult literature that suggests anesthesia may mute the effects of acupuncture (86), as well as a meta-analysis suggesting that patients must be awake rather than anesthetized in order to receive the antiemetic benefits of acupuncture (87).

Similar findings were observed in a study examining the effects of laser-stimulated acupuncture on postoperative vomiting among a sample of children undergoing strabismus surgery (79). Forty children were randomized to receive either low-level laser stimulation of acupoints at P6 $(n=20$, mean age $=5.9)$, or placebo acupuncture in which an inactive laser was held over P6 $(n=20$, mean age $=6.3)$. Laser and placebo acupuncture interventions were delivered $15 \mathrm{~min}$ prior to the induction of anesthesia, as well as $15 \mathrm{~min}$ after arrival in the recovery room. A significant difference between study groups was observed; $85 \%$ of participants in the placebo group vomited $24 \mathrm{~h}$ after surgery, while only $25 \%$ of children who received laser acupuncture vomited during that time.

Acupuncture has also been studied under outpatient conditions among children undergoing dental treatments with general anesthesia. Somri and colleagues (88) conducted a randomized prospective study of 90 children, aged 4-12 years. Their investigation employed three study groups including an ondansetron group $(n=30)$, which represented the standard of care to prevent post-operative vomiting, an acupuncture group $(n=30)$, which received bilateral needle insertions at two locations, including P6, prior to being transferred into the operating room and a placebo group $(n=30)$, which received pre-operative saline. Both the ondansetron and acupuncture groups had significantly fewer emetic episodes after discharge on the day of surgery, as compared with the placebo group, and reported higher satisfaction with anti-emetic treatment. In addition, a significantly higher proportion of children vomited during the day of or the day after surgery in the placebo group as compared with either the ondansetron or acupuncture groups. However, no significant differences were detected between the ondansetron and acupuncture groups in terms of nausea, vomiting, or overall satisfaction.

Post-operative vomiting is also prevalent following pediatric tonsillectomy procedures. One study randomly assigned 90 children, aged 4-12 years, to one of three conditions: single dose administration of ondansetron following intubation, transcutaneous electrical stimulation of surface electrodes on several acupoints prior to induction of anesthesia and again in the post-anesthesia care unit (PACU), or a no-treatment control group (78). All children were discharged from the PACU to the day surgery care unit (DSCU) prior to returning home. No significant differences in terms of number of emetic episodes were detected between the ondansetron and acupuncture groups, however, both treatments were significantly better than the control group such that they evidenced fewer emetic episodes in the DSCU and on the day of surgery. In addition, the ondansetron sample reported significantly more clinical and symptomatic side effects than either the acupuncture or control samples. Satisfaction ratings were significantly higher among the two treatment groups as compared with the control group.

A more recent study examined the use of electroacupuncture at P6 among 120 children following a tonsillectomy, aged 4-18 years (89). Participants were randomly assigned to an active acupuncture group, a sham acupuncture group, in which needles where inserted in acupoints not associated with antiemesis, or a no-needle control group. Findings revealed a significantly lower incidence of post-operative nausea and vomiting symptoms (PONV) in acupuncture group (63\%) as compared with the control group $(93 \%)$. No significant difference in PONV was observed between the sham acupuncture group (88\%) and controls (93\%). Although vomiting occurred in $63 \%$ of the acupuncture group, $88 \%$ in the sham group and $78 \%$ of the control group, these differences were not significant.

Shenkman and colleagues (80) also investigated the use of acupressure and acupuncture in children undergoing 
tonsillectomy. Forty-seven children, aged 2-12 years, were randomly assigned to receive acupressure, in the form of Acubands with adjustable straps over a spherical bead and acupuncture at P6. After anesthetic induction, but prior to glycopyrrolate or morphine administration, Acubands were replaced with intradermal needles that were covered with tape. Fifty-three patients were assigned to the control group in which sham acupoints were employed, no spherical beads were used with the Acubands, and no needles were inserted under the tape. No significant differences were found between study groups in terms of post-operative vomiting.

The use of various forms of acupuncture, including laser acupuncture, electrically stimulated acupuncture, Korean acupuncture and acupressure have been assessed in terms of their potential antiemetic prophylactic properties. Although the literature includes mixed findings, acupuncture therapies seem to result in a reduction in emetic episodes when administered prior to anesthetic induction. As noted, more research has examined post-operative vomiting as compared with other pediatric conditions; therefore, patterns of effectiveness have begun to emerge. This relationship highlights the benefits of combining the cumulative findings of large-scale, randomized, double blind and placebo-controlled studies in order to assess the empirical value of a given intervention.

\section{Cancer-related Conditions}

Pediatric patients with cancer have incorporated CAM into their treatment regimen to a relatively large extent, with prevalence rates ranging from 31 to $84 \%$ (90). However, there is much controversy over the use of these methods in the face of limited empirical research to support their efficacy, especially when families opt to employ alternative methods in lieu of conventional, evidence-based therapies (91).

Several studies have examined the use of acupuncture for the management of chemotherapy-induced nausea and vomiting, however, most tend to be among adult populations. These studies generally suggest significant reductions in nausea and vomiting among samples undergoing chemotherapy (92-95). As a result, the National Institute of Health (NIH) has approved acupuncture for the management of chemotherapyinduced nausea and vomiting (96).

Only one study specifically examined a pediatric cancer population. Reindl and colleagues (97) conducted a recent interim analysis for a randomized, crossover study among 11 children, aged 10-16 years, who were undergoing chemotherapy for the treatment of a solid tumor. Patients were randomized to receive antiemetic medication (phenothiazines) plus acupuncture, or antiemetic medication alone. Findings revealed that the use of additional antiemetic medication was reduced among participants who were also undergoing acupuncture, however, no significant differences in episodes of vomiting were detected. Personal journals kept by participants noted that continuation of acupuncture treatment was based upon self-reported reduction of chemotherapyrelated side effects, and a higher level of mental alertness as a result of acupuncture. These promising results, however, must be interpreted with caution. Acupuncture was administered at the discretion of the acupuncturist, such that no specific points were examined, but rather individually determined sites were employed for each participant.

Although acupuncture has been found efficacious among adult oncology samples for chemotherapy-induced nausea and vomiting, more research is required before such claims can be made among pediatric populations. While Whitsett and colleagues (91) warn against the use of untested therapies, they highlight the power of hope among children and families faced with cancer. As a result, continued efforts must be made in order to augment the sense of hope that appears to accompany most CAM treatments with a strong empirical basis demonstrating efficacy and safety.

\section{Discussion}

Acupuncture has been investigated as a potential treatment for a diverse range of medical conditions and demonstrates relative promise in the management of pediatric pain $(37,39)$, migraines (49), infant distress (50), enuresis (55-58), constipation (62), allergies (67), neurologic disability $(68,69,73,76)$, laryngospasm (77) and post-operative vomiting $(78,79,84)$. However, some inconsistent findings have been reported among pediatric populations $(64,80,81,85)$, suggesting the need for additional, methodologically sound research in order to assert acupuncture as an empirically valid intervention for childhood conditions. More specifically, several methodological barriers and conventional fears have contributed to the relative dearth of empirically sound studies. The limited scientific literature investigating acupuncture may result from the patient-specific needling inherent in the practice of acupuncture, small sample sizes, a lack of control groups, the role of the placebo effect (98), as well as initial problems with funding, resources and institutional support. Nonetheless, children and their families continue to seek these services, even in the face of limited findings.

In Western Medicine, treatment regimens tend to be standardized, however, the tenets of Traditional Chinese Medicine suggest an individualized approach to patient care. Therefore, the practice of acupuncture is largely dictated by the specialization of the practitioner and the needs of the patient. Filshie and Cummings (98) note areas of variability, including acupoint selection, number of needles per session, needle diameter, depth of insertion 
and duration of needling. Therefore, the variability inherent to the implementation of acupuncture makes it difficult to examine through standard methodological approaches.

Likewise, most acupuncture literature is generated in adult populations in China under a more lenient methodological framework. Reports tend to be descriptive in nature with unclear outcome measures often reported as 'cured', 'greatly improved', or 'slightly improved' (98-100). In addition, most studies are retrospective, do not include control groups, employ undefined inclusion and exclusion criteria, and do not report statistical analyses. While this research may provide a qualitative review of acupuncture, it is difficult to rely on these reports as an unbiased endorsement of effectiveness. Future acupuncture research should be based on rigorous study methodology and comparison with a randomized control group, such as a wait list, standard care, alternative treatment, or sham acupuncture group.

Alternatively, investigators in pain management have discussed the utility of practical controlled trials (PCTs) versus randomized controlled trials (RCTs). PCTs are designed to answer specific questions faced by health care providers and decision makers, such as those regarding the costs, benefits, safety, or feasibility of a particular intervention (101). They are particularly well suited for the study of complex interventions, such as CAM therapies, as they assess traditional outcome measures as well as the environment in which a therapy is delivered and its influences on broad health outcomes, such as quality of life (102). While the RCT is often limited by strict inclusion/exclusion criteria, the PCT assesses a broad range of health outcomes across diverse populations and practice settings. An example of a PCT may be a natural comparison of yoga, acupuncture and pharmaceutical management for low back pain in participants from diverse backgrounds (103). This PCT study would examine pain outcomes as well as quality of life outcomes and outside healing influences.

In addition to understanding the methodological limitations associated with the pediatric acupuncture literature base, one must address concerns related to potential side effects, and the acceptability of this treatment among parents and children. Research has consistently shown that the occurrence of adverse events in the practice of acupuncture is rare (44). Although there have been case reports of acupuncture-associated fatal pneumothoraces (104), cardiac tamponade (105) and serious infections (106), the reported incidence of serious side effects from acupuncture therapy performed by a licensed acupuncturist ranges from 1 in 10000 to 1 in $100000(15,107,108)$. In a review of first-hand accounts of acupuncture-related complications and adverse effects, Lao and colleagues (109) identified only 202 reported cases in 35 years of literature.
While serious consequences of acupuncture are rare, adverse effects can occur (110). Several studies report injuries to the central nervous system, including direct injury to the spinal cord and the spinal nerve roots, as well as indirect injury by needle fragments $(111,112)$. A Swedish study observed bleeding in 1371 of 9277 (14.8\%) acupuncture treatments, as well as transient symptoms, including faintness, sweating and fatigue (113). A large Australian survey demonstrated that local skin infections occurred after acupuncture in 128 of 3222 cases (114). Likewise, though the chances of serious infection are quite low, there are several case reports suggesting that hepatitis, HIV and sub acute bacterial endocarditis may develop after acupuncture treatment (115-117).

Improper handling of the acupuncture needles or reuse without proper sterilization can increase the risk of infections (108). Consequently, the NIH Consensus Developmental Panel on Acupuncture (96) calls for appropriate safeguards to be implemented for the protection of patients. Acupuncture needle use must also follow FDA regulations, including the use of sterile, single-use needles. In addition, access to licensed and certified acupuncture professionals should be ensured for all patients.

In contrast to conventional thought that children are afraid of needles, studies suggest that most children tolerate acupuncture therapies well and view acupuncture as a positive experience (37-39). However, as some children may still be wary of acupuncture treatment, many pediatric acupuncturists use gentle or non-invasive treatments such as low-level electrical stimulation, magnets, press balls, indirect moxabustion and warming skin salve to stimulate acupuncture points without penetrating the skin (1).

As the scientific literature investigating the role of acupuncture in pediatric populations continues to grow, health care professionals must be prepared to discuss complementary and alternative interventions with families. However, a significant amount of additional research is still needed in order to establish a sound empirical basis for the incorporation of acupuncture into standard care. Given the promising trends in the current research, the relative willingness of families to engage in acupuncture and the low risk of deleterious side effects, acupuncture may serve to harmonize Traditional Western Medicine and Traditional Chinese Medicine as a means of promoting preventive care and symptom management for children.

\section{References}

1. Lee AC, Highfield ES, Berde CB, Kemper KJ. Survey of acupuncturists: practice characteristics and pediatric care. West $J$ Med 1999;171:153-7.

2. Bellas A, Lafferty WE, Lind B, Tyree PT. Frequency, predictors, and expenditures for pediatric insurance claims for complementary 
and alternative medical professionals in Washington State. Arch Pediatr Adolesc Med 2005;159:367-72.

3. Hughes A, Penner M. Reimbursement for complimentary/ alternative medicine by California HMOs. Manag Care $Q$ 2001;9:1-4.

4. Davis MP, Darden PM. Use of complementary and alternative medicine by children in the United States. Arch Pediatr Adolesc Med 2003; 157:393-6.

5. Ottolini MC, Hamburger EK, Loprieato JO, Coleman RH, Sachs HC, Madden R, et al. Complementary and alternative medicine use among children in the Washington, DC area. Ambul Pediatr 2001:1:122-5.

6. Simpson N, Pearce A, Finlay F, Lenton S. The use of complementary medicine in paediatric outpatient clinics. Ambul Child Health 1998;3:351-6.

7. Grootenhuis MA, Last BF, de Graaf-Nijkerk JH, van der Wel M. Use of alternative treatment in pediatric oncology. Cancer Nurs 1998;21:282-8.

8. Stern RC, Canda ER, Doershuk CF. Use of nonmedical treatment by cystic fibrosis patients. J Adolesc Health 1992;13:612-5.

9. National Center for Complementary and Alternative Medicine. NCCAM Funding: Appropriations History (Accessed on February 26, 2007). Available at: http://nccam.nih.gov/about/ appropriations

10. Berman BM, Lao L, Greene M, Anderson RW, Wong RH, Langenberg P, et al. Efficacy of traditional Chinese acupuncture in the treatment of symptomatic knee osteoarthritis: a pilot study. Osteoarthr Cartil 1995;3:139-42.

11. Drivdahl C, Miser W. The use of alternative health care by a family practice population. J Am Board Fam Med 1998;11:193-9.

12. Spigelblatt L, Laine-Ammara G, Pless IB, Guyver A. The use of alternative medicine by children. Pediatrics 1994;94:811-4

13. Diehl DL, Kaplan G, Coulter I, Glik D, Hurwitz EL. Use of acupuncture by American physicians. J Altern Complement Med 1997:3:119-26.

14. Verhoef MJ, Sutherland LR. Alternative medicine and general practitioners. Opinions and behaviour. Can Fam Physician 1995;41:1005-11.

15. White A, Resch K, Ernst E. A survey of complementary practitioners' fees, practice and attitudes to working within the National Health Service. Complement Ther Med 1997;5:210-4.

16. Boyd D. Traditional Chinese medicine and cancer: an integrative approach. Presented at UCLA Center for Integrative Medicine, Los Angeles, CA, January 14, 2003.

17. Sionneau P, Gang L. The Treatment of Disease in TCM Vol. 5 Chest and Abdomen. Boulder, CO: Blue Poppy Press, 1999.

18. Maciocia G. Obstetrics \& Gynecology in Chinese Medicine. New York, NY: Churchill Livingstone, 1998.

19. O'Connor J, Bensky D. Acupuncture, a comprehensive text. Seattle, WA: Eastland Press, 1981.

20. Matsumoto K, Euler D. Kiiko Matsumoto's Clinical Strategies: In the Spirit of Master Nagano, Vol. 1. Natick, MA: Kiiko Matsumoto International, 2002.

21. Yang SZ, Li JY. Li Dong Yuan's Treatise on the Spleen and Stomach. Boulder, CO: Blue Poppy Press, 1993.

22. Breuner CC. Complementary medicine in pediatrics: a review of acupuncture, homeopathy, massage, and chiropractic therapies. Curr Probl Pediatr Adolesc Health Care 2002;32:353-84.

23. Vickers A, Zollman C. ABC of complementary medicine. Acupuncture. BMJ 1999;319:973-6.

24. Sims J, Sung J, Liao M, Lee K. The mechanisms of acupuncture analgesia: a review. Complement Ther Med 1997;5:102-11.

25. Martin DP, Sletten CD, Williams BA, Berger IH. Improvement in fibromyalgia symptoms with acupuncture: results of a randomized controlled trial. Mayo Clin Proc 2006;81:749-57.

26. Singh BB, Wu WS, Hwang SH, Khorsan R, Der-Martirosian C, Vinjamury SP, et al. Effectiveness of acupuncture in the treatment of fibromyalgia. Altern Ther Health Med 2006;12:34-41.

27. Pan CX, Morrison RS, Ness J, Fugh-Berman A, Leipzig RM. Complementary and alternative medicine in the management of pain, dyspnea, and nausea and vomiting near the end of life. A systematic review. J Pain Symptom Manage 2000;20:374-87.

28. Ezzo J, Lao L, Berman BM. Assessing clinical efficacy of acupuncture: what has been learned from systematic reviews of acupuncture?. In: Stux G, Hammerschlag R (eds), Clinical Acupuncture: Scientific Basis. New York, NY: Springer, 2001, $113-30$.

29. Vickers A, Wilson P, Kleijnen J. Acupuncture. Qual Saf Health Care 2002;11:92-7.

30. Park J, White AR, Ernst E. Efficacy of acupuncture as a treatment for tinnitus: a systematic review. Arch Otolaryngol Head Neck Surg 2000; 126:489-92.

31. Birch S. An overview of acupuncture in the treatment of stroke, addiction and other health problems. In: Stux G, Hammerschlag R (eds),. Clinical Acupuncture: Scientific Basis. New York, NY: Springer, 2001, 131-49.

32. Wang SM, Kain ZN. Auricular acupuncture: a potential treatment for anxiety. Anesth Analg 2001;92:548-53.

33. Wang SM, Peloquin C, Kain ZN. The use of auricular acupuncture to reduce preoperative anxiety. Anesth Analg 2001;93:1178-80.

34. Porzio G, Trapasso T, Martelli S, Sallusti E, Piccone C, Mattei A, et al. Acupuncture in the treatment of menopause-related symptoms in women taking tamoxifen. Tumori 2002;88:128-30.

35. Ezzo J, Berman B, Hadhazy VA, Jadad AR, Lao L, Singh BB. Is acupuncture effective for the treatment of chronic pain? A systematic review. Pain 2000;86:217-25.

36. Lin Y, Lee A, Kemper K. Acupuncture services provided by pediatric pain treatment services in North America. Pediatric Academic Society Meeting Abstract, May 1999.

37. Kemper KJ, Sarah R, Silver-Highfield E, Xiarhos E, Barnes L, Berde C. On pins and needles? Pediatric pain patients' experience with acupuncture. Pediatrics 2000;105:941-7.

38. Tsao JC, Meldrum M, Bursch B, Jacob MC, Kim SC, Zeltzer LK. Treatment expectations for CAM interventions in pediatric chronic pain patients and their parents. Evid Based Complement Alternat Med 2005;2:521-7.

39. Zeltzer LK, Tsao JC, Stelling C, Powers M, Levy S, Waterhouse M. A phase I study on the feasibility and acceptability of an acupuncture/hypnosis intervention for chronic pediatric pain J Pain Symptom Manage 2002;24:437-46.

40. Varni JW, Thompson KL, Hanson V. The Varni/Thompson pediatric pain questionnaire I. Chronic musculoskeletal pain in juvenile rheumatoid arthritis. Pain 1987:28:27-38.

41. Kovacs M. Children's Depression Inventory Manual. North Tonawanda, NY: Multi-Health Systems, 1992.

42. Spielberger CS, Gorsuch RL, Lushene RE. Manual for the State Trait Anxiety Inventory. Palo Alto, CA: Consulting Psychologists Press, 1973.

43. Lin Y, Bioteau AB, Lee AC. Acupuncture for the management of pediatric pain: a pilot study. Acupunct Med 2003;14:45-6.

44. Lin Y, Ly H, Bioteau AB. Acupuncture in the management of chronic fatigue syndrome in adolescents: a pilot study. Acupunct Med 2004;16:21-3.

45. Jodorkovsky R. Hand acupuncture experience in pediatric patients. Acupunct Med 1999;11:25-8.

46. Melchart D, Linde K, Fischer P, Berman B, White A, Vickers A, et al. Acupuncture for idiopathic headache. Cochrane Database Syst Rev 2001;1:765-79.

47. Vincent CA. The treatment of tension headache by acupuncture: a controlled single case design with time series analysis. $J$ Psychosom Res 1990;34:553-61.

48. Pintov S, Lahat E, Alstein M, Vogel Z, Barg J. Acupuncture and the opioid system: implications in management of migraine. Pediatr Neurol 1997;17:129-33.

49. Schwartz L, Bauchner H, Blocleer R, Jorgensen K, Pearson C, O'Donnella R, et al. Salivary cortisol as an indicator of stress in premature infants: the effect of electrical stimulation of acupuncture meridians in blunting this response. Acupunct Med 1999;10:27-30.

50. Baoqin X. 302 cases of enuresis treated with acupuncture. J Tradit Chin Med 1991;11:121-2.

51. Ionescu-Tirgoviste C, Visinescu R, Ionescu C, Tomescu M. The treatment of enuresis by acupuncture. Am J Acupunct 1983;11:119-24.

52. Roje-Starcevic M. The treatment of nocturnal enuresis by acupuncture. Neurologija 1990;39:179-84.

53. Tuzuner F, Kecik Y, Ozdemir S, Canakci N. Electro-acupuncture in the treatment of enuresis nocturna. Acupunct Electrother Res 1989;14:211-5. 
54. Bower WF, Diao M, Tang JL, Yeung CK. Acupuncture for nocturnal enuresis in children: a systematic review and exploration of rationale. Neurourol Urodyn 2005;24:267-72.

55. Radmayr C, Schlager A, Studen M, Bartsch G. Prospective randomized trial using laser acupuncture versus desmopressin in the treatment of nocturnal enuresis. Eur Urol 2001;40:201-5.

56. Bjorkstrom G, Hellstrom AL, Andersson S. Electro-acupuncture in the treatment of children with monosymptomatic nocturnal enuresis. Scand J Urol Nephrol 2000;34:21-6.

57. Honjo H, Kawauchi A, Ukimura O, Soh J, Mizutani Y, Miki T. Treatment of monosymptomatic nocturnal enuresis by acupuncture: a preliminary study. Int $J$ Urol 2002;9:672-6.

58. Serel TA, Perk H, Koyuncuoglu HR, Kosar A, Celik K, Deniz N. Acupuncture therapy in the management of persistent primary nocturnal enuresis-preliminary results. Scand J Urol Nephrol 2001;35:40-3.

59. Jodorkovsky R. Treatment of primary nocturnal enuresis with hand therapy: a randomized double-blind, placebo-controlled trial. Acupunc Med 2003;14:28-31.

60. Hamano S, Yamanishi T, Igarashi $\mathrm{T}$, Ito $\mathrm{H}$, Murakami $\mathrm{S}$. Functional bladder capacity as predictor of response to desmopressin and retention control training in monosymptomatic nocturnal enuresis. Eur Urol 2000;37:718-22.

61. Monda JM, Husmann DA. Primary nocturnal enuresis: a comparison among observation, imipramine, desmopressin acetate and bedwetting alarm systems. J Urol 1995;154:745-8.

62. Broide E, Pintov S, Portnoy S, Barg J, Klinowski E, Scapa E. Effectiveness of acupuncture for treatment of childhood constipation. Dig Dis Sci 2001;46:1270-5.

63. Cheuk DK, Wong V. Acupuncture for epilepsy. Cochrane Database Syst Rev 2006;2:CD005062.

64. Xiong X, Zhang G, Huang W, Sun J. Clinical observation of acupuncture and Chinese medicine for treatment of epilepsy in children. J Tradit Chin Med 2003;10:62-3.

65. Blanc PD, Trupin L, Earnest G, Katz PP, Yelin EH, Eisner MD. Alternative therapies among adults with a reported diagnosis of asthma or rhinosinusitis: data from a population-based survey. Chest 2001;120:1461-7.

66. Xue CC, English R, Zhang JJ, Da Costa C, Li CG. Effect of acupuncture in the treatment of seasonal allergic rhinitis: a randomized controlled clinical trial. Am J Chin Med 2002;30:1-11.

67. Ng DK, Chow PY, Ming SP, Hong SH, Lau S, Tse D, et al. A double-blind, randomized, placebo-controlled trial of acupuncture for the treatment of childhood persistent allergic rhinitis. Pediatrics 2004:114:1242-7.

68. Svedberg L, Nordahl G, Lundeberg T. Effects of acupuncture on skin temperature in children with neurological disorders and cold feet. Dev Med Child Neurol 2001:43:358-60.

69. Wong V, Sun JG, Wong W. Traditional Chinese medicine (tongue acupuncture) in children with drooling problems. Pediatr Neurol 2001;25:47-54.

70. To R, Wong V, Hui Y. Pilot study of oro-motor habilitation program for children with drooling problem: a pilot observation. HK J Paediatr 1999;4:96-100.

71. Heine RG, Catto-Smith AG, Reddihough DS. Effect of antireflux medication on salivary drooling in children with cerebral palsy. Dev Med Child Neurol 1996;38:1030-6.

72. Rapp D. Drool control: long-term follow-up. Dev Med Child Neurol 1980;22:448-53.

73. Sun J, Ko C, Wong V, Sun X. Randomised control trial of tongue acupuncture versus sham acupuncture in improving functional outcome in cerebral palsy. $J$ Neurol Neurosurg Psychiatry 2004; $75: 1054-7$

74. Russell DJ, Rosenbaum PL, Gowland C, Hardy S, Lane M, Plews N, et al. The Gross Motor Function Measure Manual, 2nd edn. Hamilton, ON: McMaster University, 1993.

75. Haley SM, Coster WJ, Ludlow LH, Haltiwanger JT, Andrellos PJ. Pediatric Evaluation of Disability Inventory (PEDI), Version 1, Development, Standardization, and Administration Manual. Boston, MA: New England Medical Center, 1992.

76. Duncan B, Barton L, Edmonds D, Blashill BM. Parental perceptions of the therapeutic effect from osteopathic manipulation or acupuncture in children with spastic cerebral palsy. Clin.Pediatr Phila) 2004;43:349-53.
77. Lee CK, Chien TJ, Hsu JC, Yang CY, Hsiao JM, Huang YR, et al. The effect of acupuncture on the incidence of postextubation laryngospasm in children. Anaesthesia 1998;53:917-20.

78. Kabalak AA, Akcay M, Akcay F, Gogus N. Transcutaneous electrical acupoint stimulation versus ondansetron in the prevention of postoperative vomiting following pediatric tonsillectomy. J Altern Complement Med 2005;11:407-13.

79. Schlager A, Offer T, Baldissera I. Laser stimulation of acupuncture point P6 reduces postoperative vomiting in children undergoing strabismus surgery. Br J Anaesth 1998;81:529-32.

80. Shenkman Z, Holzman RS, Kim C, Ferrari LR, DiCanzio J, Highfield ES, et al. Acupressure-acupuncture antiemetic prophylaxis in children undergoing tonsillectomy. Anesthesiology 1999:90:1311-6.

81. Yentis SM, Bissonnette B. Ineffectiveness of acupuncture and droperidol in preventing vomiting following strabismus repair in children. Can J Anaesth 1992;39:151-4.

82. Lerman J, Eustis S, Smith DR. Effect of droperidol pretreatment on postanesthetic vomiting in children undergoing strabismus surgery. Anesthesiology 1986:65:322-5.

83. Wang SM, Kain ZN. P6 acupoint injections are as effective as droperidol in controlling early postoperative nausea and vomiting in children. Anesthesiology 2002;97:359-66.

84. Schlager A, Boehler M, Puhringer F. Korean hand acupressure reduces postoperative vomiting in children after strabismus surgery. Br J Anaesth 2000;85:267-70.

85. Yentis SM, Bissonnette B. P6 acupuncture and postoperative vomiting after tonsillectomy in children. $\mathrm{Br} \quad J$ Anaesth 1991;67:779-80.

86. Dundee JW, Milligan KR, McKay AC. Influence of intraoperative acupuncture and droperidol on postoperative emesis. $\mathrm{Br} J$ Anaesth 1988;61:117P-8P.

87. Vickers AJ. Can acupuncture have specific effects on health? A systematic review of acupuncture antiemesis trials. $J R$ Soc Med 1996;89:303-11.

88. Somri M, Vaida SJ, Sabo E, Yassain G, Gankin I, Gaitini LA. Acupuncture versus ondansetron in the prevention of postoperative vomiting. A study of children undergoing dental surgery. Anaesthesia 2001;56:927-32.

89. Rusy LM, Hoffman GM, Weisman SJ. Electroacupuncture prophylaxis of postoperative nausea and vomiting following pediatric tonsillectomy with or without adenoidectomy. Anesthesiology 2002;96:300-5.

90. Sencer SF, Kelly KM. Bringing evidence to complementary and alternative medicine for children with cancer. $J$ Pediatr Hematol Oncol 2006;28:186-9.

91. Whitsett SF, Anderson R, Coppes MJ. Why are children with cancer being exposed to complementary medicine? West J Med 1999:171:150-1.

92. Collins KB, Thomas DJ. Acupuncture and acupressure for the management of chemotherapy-induced nausea and vomiting. $J \mathrm{Am}$ Acad Nurse Pract 2004;16:76-80.

93. Dibble SL, Chapman J, Mack KA, Shih AS. Acupressure for nausea: results of a pilot study. Oncol Nurs Forum 2000;27:41-7.

94. Dundee JW, Yang J. Prolongation of the antiemetic action of P6 acupuncture by acupressure in patients having cancer chemotherapy. J R Soc Med 1990;83:360-2.

95. Shen J, Wenger N, Glaspy J, Hays RD, Albert PS, Choi C, et al. Electroacupuncture for control of myeloablative chemotherapyinduced emesis: a randomized controlled trial. JAMA 2000;284:2755-61.

96. NIH Consensus Conference. Acupuncture. JAMA 1998;280:1518-24.

97. Reindl TK, Geilen W, Hartmann R, Wiebelitz KR, Kan G, Wilhelm I, et al. Acupuncture against chemotherapy-induced nausea and vomiting in pediatric oncology. Interim results of a multicenter crossover study. Support Care Cancer 2006;14:172-6.

98. Filshie J, Cummings TM. Western medical acupuncture. In: Ernst E, White A (eds),. Acupuncture: A Scientific Appraisal. Oxford: Butterworth Heinemann, 1999, 31-59.

99. Bingpei S, Liyu L, Huaidi B. Clinical Observation on 617 cases of pediatric syndrome of atrophic paralysis of the limbs treated with acupuncture. J Tradit Chin Med 1989;9:179-81. 
100. Bingpei S, Huaidi B, Liyu L. A clinical study on acupuncture treatment of pediatric cerebral palsy. $J$ Tradit Chin Med 1992;12:45-51.

101. Pincus T, Stein CM. Why randomized controlled clinical trials do not depict accurately long-term outcomes in rheumatoid arthritis: some explanations and suggestions for future studies. Clin Exp Rheumatol 1997;15 (Suppl 17):S27-38.

102. Rakel D. Practical controlled trials: researching the therapeutic ritual and all its parts. Aust Prosthodont Soc Bull 2006;16.

103. Caspi O. Evidenced-based medicine and clinical decision making. In: Rakel DP (ed). Integrated Medicine. Philadelphia, PA: Elsevier Medical Publisher; 2007.

104. Olusanya O, Mansuri I. Pneumothorax following acupuncture. J Am Board Fam Pract 1997:10:296-7.

105. Kataoka H. Cardiac tamponade caused by penetration of an acupuncture needle into the right ventricle. $J$ Thorac Cardiovasc Surg 1997;114:674-6.

106. Matsumura Y, Inui M, Tagawa T. Peritemporomandibular abscess as a complication of acupuncture treatment. J Oral Maxillofac Surg 1998:56:495-6.

107. MacPherson H. Fatal and adverse events from acupuncture: allegation, evidence and implications. J Altern Complement Med 1999;5:47-56.

108. Ernst E, White A. Life-threatening adverse reactions after acupuncture? Pain 1997;71:123-6.
109. Lao L, Hamilton GR, Fu J, Berman BM. Is acupuncture safe? A systematic review of case reports. Altern Ther Health Med 2003;9:72-83.

110. Niggemann B, Gruber C. Side-effects of complementary and alternative medicine. Allergy 2003;58:707-16.

111. Peuker ET, White A, Ernst E, Pera F, Filler TJ. Traumatic complications of acupuncture. Therapists need to know human anatomy. Arch Fam Med 1999;8:553-8.

112. Yamashita H, Tsukayam H, White AR, Tanno Y, Sugishita C, Ernst E. Systematic review of adverse events folowing acupuncture: the Japanese literature. Complement Ther Med 2001;9:98-104.

113. Odsberg A, Schill U, Haker E. Acupuncture treatment: side effects and complications reported by Swedish physiotherapists. Complement Ther Med 2001;9:17-20.

114. Bensoussan A, Myers SP, Carlton AL. Risks associated with the practice of traditional Chinese medicine: an Australian study. Arch Fam Med 2000;9:1071-8.

115. Rampes H, James R. Complications of acupuncture. Acupunct Med 1995;13:26-33.

116. Jeerys DB, Smith S, Brennand-Roper DA, Curry PVL. Acupuncture needles as a cause of bacterial endocarditis. $\mathrm{Br} \mathrm{Med}$ $J$ 1983;287:326-7.

117. Vittecoq D, Mettetal JF, Rouzioux C, Bach JF, Bouchon JP. Acute HIV infection after acupuncture treatments. $N$ Engl J Med 1989;320:250-1.

Received March 5, 2007; accepted November 28, 2007 


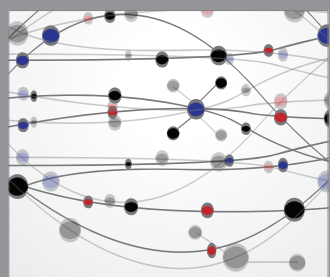

The Scientific World Journal
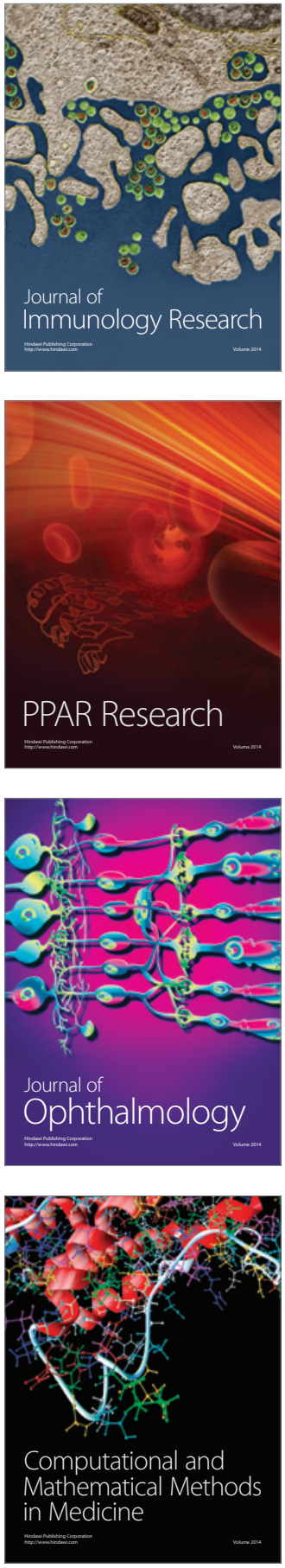

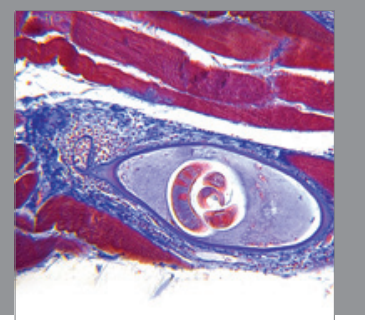

Gastroenterology

Research and Practice
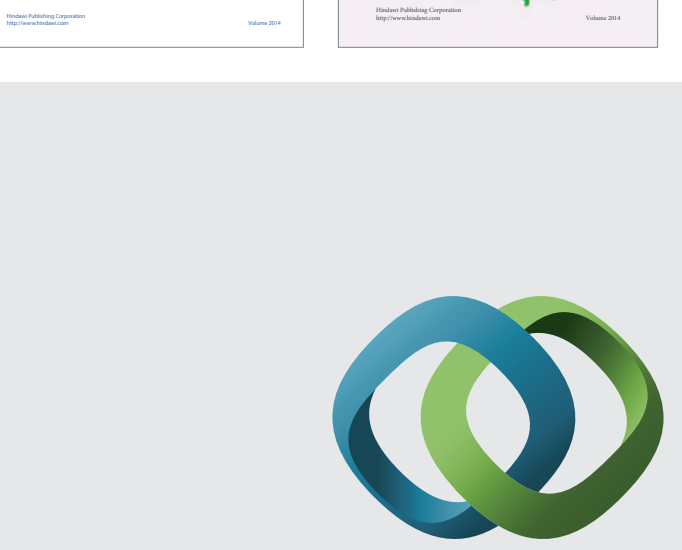

\section{Hindawi}

Submit your manuscripts at

http://www.hindawi.com
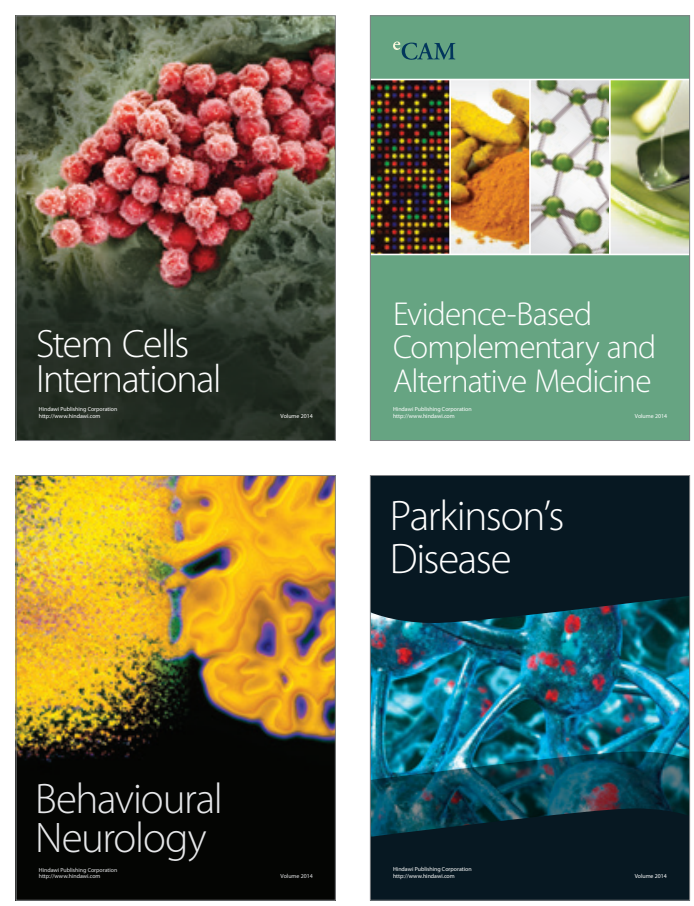

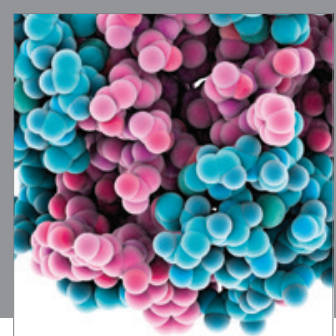

Journal of
Diabetes Research

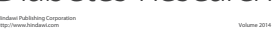

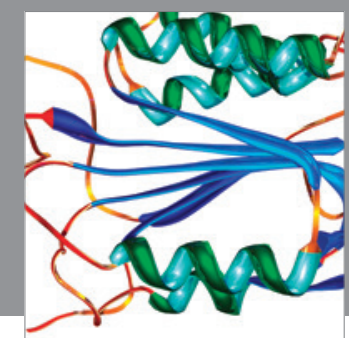

Disease Markers
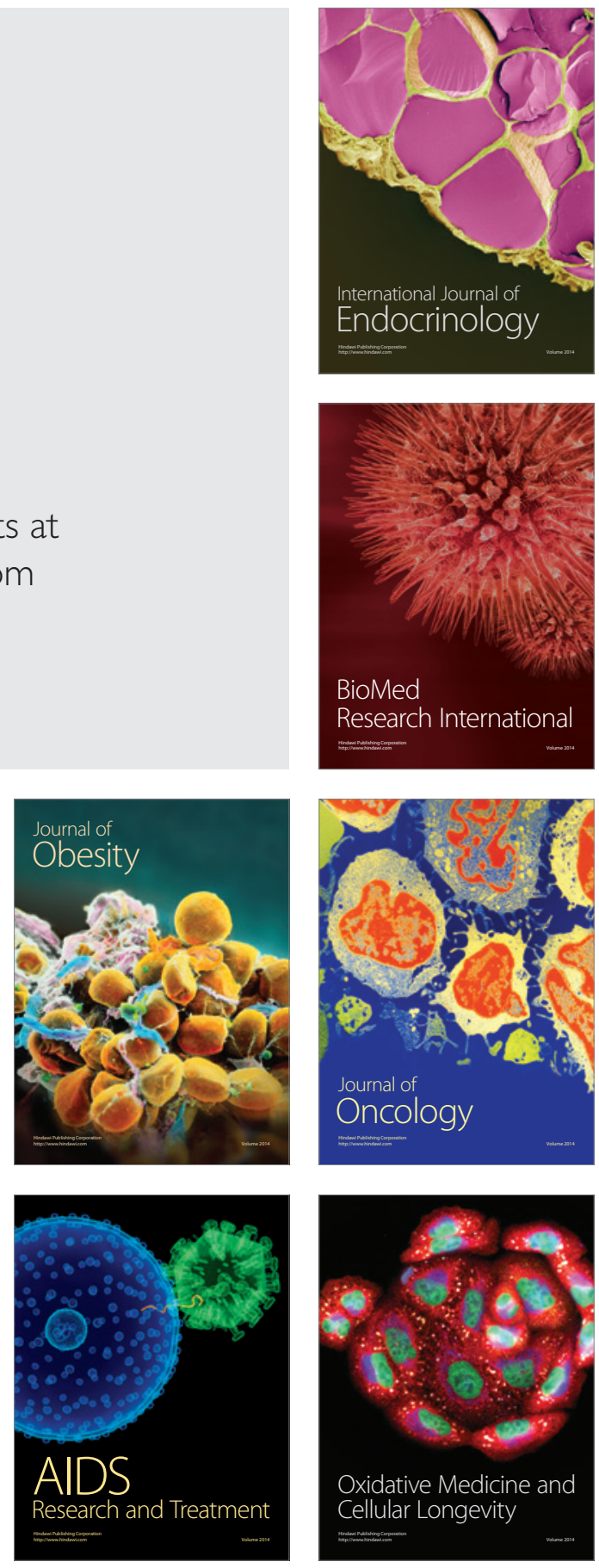